\title{
How hardiness and the quality of life relate to the parameters of autonomic balance
}

\author{
Elena Nikolaeva ${ }^{1, *}$, Oksana Elnikova ${ }^{2}$, Eugeny Vergunov $^{3}$, and Yulia Perevoskina ${ }^{4}$ \\ ${ }^{1}$ Herzen State Pedagogical University, Russian Federation \\ ${ }^{2}$ Bunin State University, Russian Federation \\ ${ }^{3}$ Scientific Research Institute of Physiology and Basic Medicine, Russian Federation \\ ${ }^{4}$ Novosibirsk State Pedagogical University, Russian Federation
}

\begin{abstract}
The purpose of the research was to evaluate the prognostic significance of the two tests most often used by psychologists for describing of the subject's quality of life: the Short Form-36 Health Status Survey (SF-36) and the Hardiness Test (Maddi, 1998). As an indicator of the state of internal control, the activity of the sympathetic nervous system (SNS) and the parasympathetic nervous system (PSNS) was chosen. Our study sample consisted of 59 students and office workers (mean age of $25.1 \pm 11.0$ years). The study was conducted in two successive stages. During each of the stages, the heart rate was recorded, the slow and the fast waves on the cardiogram were assessed. During the first stage, the R-R intervals were recorded with the subject in a state of quiet wakefulness; during the second, while the subject's health was being discussed. A second recording was done for further elaboration (once again while the test subject was in the process of describing the state of their health). The only correlation between a person's own description and the actual inner state arises when they are describing their real responsibilities and how they carry them out.
\end{abstract}

Key words: quality of life, autonomic balance, self-evaluation.

\section{Introduction}

Practically all contemporary theories that explain how the brain works are based on the idea that it does not respond to external influences, but it does anticipate changes in the ambient environment [1]. It can be presumed that it makes predictions of what is happening not only in the outside world, but also of what is transpiring in the body itself. On the one hand, these predictions manifest themselves in the reactions of systems responsible for allostasis [2] and, on the other hand, in a person's verbal description of their own state. Thus, the predictive capability of the brain with regard to the intrinsic state of a particular person can be described as the correlation between their description of themselves and the physiological changes that are taking place in their body.

A description of this correlation is significant since it will help us satisfy our need to understand to what extent we can rely on the results of screening tests designed to reveal the early symptoms of a disease in people who have no clinical manifestations of that disease.

It can be supposed that a person's subjective description of their state of health and their attitude towards life's problems are connected in a way that is not entirely apparent with

\footnotetext{
* Corresponding author: klemtina@yandex.ru
}

(C) The Authors, published by EDP Sciences. This is an Open Access article distributed under the terms of the Creative Commons Attribution License 4.0 (http://creativecommons.org/licenses/by/4.0/). 
the peculiarities of internal control over physiological processes and therefore has prognostic value when it comes to assessing a person's health in general, as well as very early symptoms of changes in their body's regulatory processes.

It was of interest to evaluate the prognostic significance of the two tests most often used by psychologists: the Short Form-36 Health Status Survey (SF-36) and the Hardiness Test $[3,4]$. As an indicator of the state of internal control, the correlative activity of two parts of the autonomic nervous system (ANS) - the sympathetic nervous system (SNS) and the parasympathetic nervous system (PSNS) - was chosen.

The SNS and the PSNS are responsible for ensuring that different aspects of the body's internal environment function properly. While the SNS is activated when circumstances in the external environment change abruptly, the PSNS, to a great extent, sees to the recovery of spent resources. It is for this reason that, when the body's level of activity is low, these systems may even function uni-directionally, whereas, at the height of its functioning capacity, their activities are orthographic and aimed in different directions. And very often the activity of one system suppresses that of the other.

Activation of the SNS attests to the degree of the allostatic load in the trial subject and corresponds to the stress level of the regulatory systems [5, 6]. Activation of the parasympathetic nervous system, which originated earlier in evolution than the sympathetic system to provide stability within the internal environment, demonstrates the level of the body's reserve possibilities.

The SF-36 gauges the quality of life according to eight scales [7]. It is widely used to assess the condition of people who are ill [8]. But it has the potential to be used in screening those who are essentially healthy. Scales 1-4 portray the "physical component of health", and Scales 5-8 provide a picture of the "psychological component".

Hardiness represents a system of belief in the self, the world and the relationship between the two. It is a mode of functioning that includes three relatively autonomous components: the dispositions of commitment, control and challenge. According to the authors of this paper, manifestation of these three components, and of hardiness on the whole, safeguards the body against internal pressure in stressful situations by means of hardy coping with the stress and downplaying of its significance. S.R. Maddi [3, 4] emphasizes how important these three components are for the maintenance of health, as well as an optimum level of working capacity and alertness under stressogenic conditions.

It can be assumed that, among those people who are healthy, hardiness and their own conception of their state of health will be reflected by changes in the balance between the sympathetic and the parasympathetic branches of the autonomic nervous system, evaluated during the states of quiet wakefulness and of stress - in this case, during a conversation with them about their health. Their reaction to this conversation will depend on the person's own assessment of the state of their health and how they feel about it. Therefore, the intensity of the changes in the balance between the SNS and the PSNS can reflect a person's view of themselves as either healthy or unhealthy.

\section{Material and methods}

Our study sample consisted of 59 students and office workers between the ages of 18 and 70 in the city of Yelets (with a mean age of $25.1 \pm 11.0$ years, hereinafter referred to as $[\mathrm{m}]+[\mathrm{SD}]$. Their heart rates were recorded by using the Omega $\mathrm{M}$ hardware and software package (The Dinamika Company, Russia) and standard leads (in sitting position, with electrodes placed on the wrists of both hands).

The study was conducted in two successive stages and in one sitting. During each of the stages, the heart rate was recorded at an RR interval of 300 , which made it possible to 
assess the slow and the fast waves on the cardiogram. During the first stage, the RR intervals were recorded with the subject in a state of quiet wakefulness; during the second, while the subject's health was being discussed. Only those test subjects who had a sinoatrial heart rate when they were in a state of quiet wakefulness participated in the study. A second recording was done for further elaboration (once again while the test subject was in the process of describing the state of their health). Technical artifacts (if there were any) were edited in Omega M software by manually adjusting erroneous markers of R-peaks into the right position. Isolated physiological artifacts (if there were any) were edited using algorithms of the Kubios HRV-heart rate variability analysis software [9].

Assessment of ANS activity was carried out based on an analysis of the subjects' heart rates, using the Nerve-Express system [10]. This software evaluates the status of the ANS on a plane, with the orthogonal axes being the parasympathetic nervous system (PSNS, the X axis) and the sympathetic nervous system (SNS, the Y axis), taking into account individual border lines for the power of the parasympathetic and sympathetic waves within the spectrum of the heart rate [10]. Negative values of the PSNS and SNS figures show a decrease in activity sharp (-4), significant $(-3)$, moderate $(-2)$ and slight $(-1)$ - corresponding to the branch of the ANS.

A zero value indicates that the system is balanced. Positive values point to an increase in activity: slight $(+1)$, moderate $(+2)$, significant $(+3)$ and sharp $(+4)$. Thus, there are 81 dots on the plane, corresponding to the ANS states received from analysis of over 10,000 test subjects. These dots are grouped in zones describing the functional state of the ANS. For example, the square at the center of the Y-axis including the PSNS and SNS values from -1 to +1 describes the zone of balanced ANS activity [10].

The Short Form-36 Health Status Survey (SF-36) was used in a version adapted for a Russian-language audience and prepared by The Evidence CPR (Clinical Pharmacology Research) Company. The scores for each scale range from 0 percent to 100 percent, with 0 percent representing a person's complete inability to carry out day-today activities on their own and 100 percent reflecting a total lack of complaints (limitations) under the weight of those same daily activities.

1. Physical Functioning (PF) reflects the degree to which a person's physical condition restricts their ability to perform physical tasks (looking after their personal needs, walking, climbing stairs, carrying heavy objects, etc.). Low scores on this scale indicate that a patient's physical activity is severely limited by the state of their health.

2. Role-Physical Functioning (RP) reflects the influence that a person's physical condition has on the roles that they fulfill in their daily activities (at work and while carrying out their everyday responsibilities). Low scores on this scale attest to a patient's daily activities being significantly limited by their physical condition.

3. Bodily Pain (BP) reflects the influence that pain syndromes have on a person's ability to engage in their day-to-day activities, including work around the home and outside of the home. Low scores here show that the pain substantially restricts a patient's activity.

4. General Health $(\mathrm{GH})$ reflects a person's own evaluation of their state of health at the present moment. The lower the score on this scale, the lower a patient's estimation of the state of their health.

5. Vitality (VT) reflects the feeling of a person that they are at full strength and energy or, on the contrary, completely drained of both. Low scores bear witness to a patient's fatigue and a lowering of their involvement in life's activities.

6. Social Functioning (SF) reflects the degree to which a person's physical or emotional state limits their social activity (interaction with other people). Low scores indicate a 
considerable limitation in a patient's social contacts and a reduced level of interaction owing to a deterioration of their physical and emotional condition.

7. Role-Emotional Functioning (RE) reflects an evaluation of the extent to which a person's emotional state interferes with their ability to work or carry out other daily activities (including large expenditures of time, a decrease in the amount of work, a lowering of its quality, etc.). Low scores on this scale are interpreted as restrictions in a patient's ability to perform their workaday activities caused by a worsening in their emotional state.

8. Mental Health $(\mathrm{MH})$ reflects the level of positive emotions. Low scores attest to the presence of mental problems, as well as depression and anxiety.

As for the Hardiness Test [3, 4], a Russian adaptation prepared by Leontyev and Rasskasova [11] was used. It has the following scales:

1. Commitment is defined as "the belief that involvement in what is happening gives the maximum chance of finding something worthwhile and interesting" (Maddi, 1998b). A person with a developed sense of commitment derives pleasure from their own activity. In contrast, the absence of such a belief gives rise to a sense of rejection and a feeling of being "outside of life". "If you feel self-confident and that the world is rather benign, you have commitment" [3, p.103].

2. Control is the conviction that the struggle allows you to have an influence on what is going on around you, even though this influence is not absolute, nor is success guaranteed. The opposite of this is the feeling of your own helplessness. A person with a highly developed sense of control feels that they choose their own sphere of action, their own way in life.

3. Challenge is the certainty a person has that everything that happens to them contributes to their development due to the knowledge that comes from experience - both positive and negative. A person who looks at life as a means of acquiring experience is ready to act in the absence of any reliable guarantees of success, at their risk and peril, believing that to strive for simple comfort and security would impoverish their life. What lies at the basis of risk-taking is the idea that development comes from the active assimilation of knowledge gained through experience and its subsequent use.

The consistency of the scales used on the study sample in the two methods (their internal validity, described with the help of Cronbach's coefficient) was within standard values.

Two statistical packages - G*Power version 3 and IBM SPSS Statistics version 22 were used to process the results. The level of statistical significance $\mathrm{p}$ was arrived at by initially assigning the value Power $=1-\beta=0.80$, and the null hypothesis deviated at $p \leq \alpha=0.05[12]$.

\section{Results and discussion}

A correlation analysis between the scores of the two questionnaires and the values of the ANS parameters was carried out. An interconnection (high, significant correlation of moderate strength) was established only between the PSNS and RP (Role-Physical Functioning) scores (Table 1). It should be noted that the other three physical components of a person's health are determined exclusively on the basis of a test subject's self-evaluation, i.e., how they assess the level of their own physical condition and physical activity as well as the level of pain that they feel. These scales merely reproduce the test subject's notions about the given actions and situations. Such self-evaluations may reflect the actual level of the body's functional reserves 
Table 1. The correlations between parameters of the quality of life and the parameters of autonomic balance (PSNS FX1 SNS).

\begin{tabular}{|l|c|c|}
\hline Scales & Parasympathetic activity & Sympathetic activity \\
\hline $\mathrm{PF}$ & $r=+0.270 ; r^{2}=0.073 ; p=0.195>0.05$ & $r=+0.162 ; r^{2}=0.026 ; p=0.338>0.05$ \\
\hline $\mathrm{RP}$ & $\boldsymbol{r}=+\mathbf{0 . 4 1 5} ; \boldsymbol{r}^{2}=\mathbf{0 . 1 7 2} ; \boldsymbol{p}=\mathbf{0 . 0 1 0}<\mathbf{0 . 0 5}$ & $r=-0.150 ; r^{2}=0.022 ; p=0.373>0.05$ \\
\hline $\mathrm{BP}$ & $r=+0.007 ; r^{2}<0.001 ; p=0.800>0.05$ & $r=+0.145 ; r^{2}=0.021 ; p=0.388>0.05$ \\
\hline $\mathrm{GH}$ & $r=+0.184 ; r^{2}=0.034 ; p=0.276>0.05$ & $r=-0.134 ; r^{2}=0.018 ; p=0.422>0.05$ \\
\hline $\mathrm{VT}$ & $r=+0.003 ; r^{2}<0.001 ; p=0.794>0.05$ & $r=-0.142 ; r^{2}=0.020 ; p=0.397>0.05$ \\
\hline $\mathrm{SF}$ & $r=+0.251 ; r^{2}=0.063 ; p=0.127>0.05$ & $r=-0.022 ; r^{2}<0.001 ; p=0.750>0.05$ \\
\hline $\mathrm{RE}$ & $r=-0.218 ; r^{2}=0.048 ; p=0.193>0.05$ & $r=+0.038 ; r^{2}=0.001 ; p=0.709>0.05$ \\
\hline MH & $r=+0.016 ; r^{2}<0.001 ; p=0.764>0.05$ & $r=+0.135 ; r^{2}=0.018 ; p=0.419>0.05$ \\
\hline
\end{tabular}

Table 2. Linear regression coefficients for PSNS.

\begin{tabular}{|l|c|c|c|c|}
\hline Coefficients & $\mathbf{B}^{*}$ & $\mathbf{m B}^{*}$ & $\boldsymbol{\beta}^{* *}$ & $\boldsymbol{\beta}^{\mathbf{*} *}$ \\
\hline Regression equation constant & -0.979 & 0.535 & & \\
\hline Age & -0.041 & 0.013 & -0.337 & 0.114 \\
\hline Role-Physical Functioning (RP) & +0.021 & 0.005 & +0.482 & 0.232 \\
\hline
\end{tabular}

Note: $*$ - nonstandard coefficients, ** - standard coefficients, $\mathrm{mB}$ - standard mistake of B coefficient.

(which are expressed in the level of parasympathetic activity for normally developing test subjects), but then again they may not reflect it $[13,14]$.

The RP scale differs from the PF, BP and GH scales in that the evaluation includes an external factor: the responsibilities that the test subject assumes as part of their professional, family and social activities. Thus, this scale can be considered a marker of how well a test subject has adapted to the society [7], which should to a certain degree correspond to the level of functional reserves in their body. Please note that there is no significant correlation with the activity of the sympathetic system, which expresses the level of stress for test subjects whose development is in keeping with the norms.

These findings conform to those of other authors. The prophetic significance of the level of parasympathetic activity in evaluating the functional state of the body when adapting to psycho-physiological workloads has been shown [10] for test subjects of various groups and ages and for different kinds of workloads.

An interdependency (a significant, weak correlation) was established only between hardiness and the RE score (the amount of work performed and the scope of daily responsibilities that are allowed by the current emotional conditions) (Table 1). There is, however, no significant correlation between hardiness and the level of ANS activity. Among the indicators of the psychological component of health, the RE indicator has distinctive features analogous to those of the RP indicator among the physical components of health [7]. It can be presupposed that if a test subject has a high level of commitment, control and challenge when involved in the life's work that they have chosen for themselves, with all other conditions being equal, this gives them an advantage in coping with the situation when there are emotional circumstances.

Thus, a person's own feeling about the state of their health is not connected with the important parameters that describe the state of the internal environment. The only correlation between a person's own description and the actual inner state arises when they are describing their real responsibilities and how they carry them out.

Regression analysis for 13 parameters (PSNS, SNS, age, sex, $8^{\text {th }}$ scales of quality of life and hardiness) shows significant correlation between PSNS and RP (Table 2). It has shown inverse correlation between PSNS and age. 


\section{Conclusion}

There is a substantial gap between a person's actual physiological reserves and the picture that they have of their own state of health, which may have evolutionary significance. This is exactly what allows a weak woman (or animal) to protect their young in a stressful situation, regardless of the body's real reserves, or a man to defend his home, or any person, no matter the gender, to commit heroic acts of survival, independent of their bodily reserves at that moment.

This situation, however, might have a negative effect when, in the absence of any threat of bodily harm, a person could, due to certain conditions and personal qualities, look for signs of a deterioration of their health - not in real physiological symptoms, but in their own imaginary notions.

The work was supported by Russian Fund of Fundamental research 17-06-00166.

\section{References}

[1] Ch. Frith, Making up the mind. How the brain creates our mental world (Singapore, Blackwell Publishing, 2011)

[2] P. Sterling, Allostasis, homeostasis, and the costs of physiological adaptation, edited by C. Schulkin (Cambridge University Press, Cambridge, UK, 2003), p. 17

[3] S.R. Maddi, Health promotion evaluation, edited by J.P. Opatz (National Wellness Institute, Stevens Point, WI, 1997), p. 101

[4] S.R. Maddi, Encyclopedia of Mental Health, edited by H.S. Friedman (Academic Press, San Diego, CA, 1998), p. 323

[5] R.M. Sapolsky, Science 308, 648 (2005)

[6] S.G. Krivoshekov, N.K. Belisheva, E.I. Nikolaeva, E.G. Vergunov, O.E. Elnikova, Humanecology 7, 17 (2016)

[7] J.E. Ware, K.K. Snow, M. Kosinski, B. Gandek, Manual and interpretation guide (Lincoln, RI, QualityMetric Inc. 2000)

[8] J.E. Ware, Arch. Phys. Med. Rehabil. 84, 43 (2003)

[9] M.P. Tarvainen, J.P. Niskanen, J.A. Lipponen, Comput. Meth. Progr. Biomed. 113(1), $210(2014)$

[10] L. Tereshchenko, S.A. Doronina, E.M. Pochinok, A. Riftine, Pac. Clin. Electrophys. 26, 444 (2003)

[11] D.A. Leontjev, E.I. Rasskasova (Hardiness test, M., Smisl., 2006)

[12] F. Faul, E. Erdfelder, A.G. Lang, A. Buchner, Behav. Res. Methods. 39(2), 175 (2007)

[13] E.I. Nikolaeva, E.G. Vergunov, A.V. Dobrin, CEUR Workshop Proceedings 1419, 521 (2015)

[14] E. Nikolaeva, Med. Psych. in Russia 1(30), 5 (2015) 